\title{
Why going organic is good
}

\author{
Organic semiconductors could one day be used in new types of spintronic devices. Before realistic \\ applications can be achieved however, more experimental and theoretical work is needed to understand \\ the mechanism of spin injection and spin transport .
}

It is not uncommon for a whole research field to originate from serendipity, and this is the case for organic spintronics, which is the topic that we focus on in this issue. The idea of injecting spin-polarized electrons into organic semiconductors and controlling their properties in spintronic devices derives from an attempt to improve the efficiency of organic light-emitting diodes (OLEDs).

In OLEDs, a large portion of electrons and holes form triplet spin states instead of the radiative singlets, which limits the light-emission efficiency. About ten years ago, Alek Dediu and Carlo Taliani combined their respective expertise in oxides and OLEDs to find ways to solve this problem. They decided to inject spinpolarized carriers into an organic layer from a ferromagnetic electrode as a means of improving the singlet/triplet ratio. Although their original project was not successful, they measured a large magnetoresistance, and in 2002 they published the first results about spin-polarized electron injection from $\mathrm{La}_{0.7} \mathrm{Sr}_{0.3} \mathrm{MnO}_{3}$ into the organic compound 6T (ref. 1). Two years later, other groups observed the spin-valve effect in a hybrid inorganic-organic device ${ }^{2}$ and the intrinsic resistance variation in an organic semiconductor when a small magnetic field is applied - a phenomenon that was termed organic magnetoresistance (OMAR) ${ }^{3}$. These results confirmed the potential of organic semiconductors for spintronics.

More experimental and theoretical studies - which are reviewed in the Progress Article by Dediu and coauthors in this issue $e^{4}$ - followed the first results. But organic semiconductors, having low mobility and complex transport properties, may seem to be far from an obvious choice for spintronics. A clear advantage they offer, particularly for devices based on spin transport, is that the spin-orbit interaction is usually very weak, resulting in long spin-relaxation times. From a practical point of view, as Valy Vardeny explains in our interview, the materials are cheap and versatile $e^{5}$. In addition, the injection of spin-polarized carriers could be used to control the light emitted by OLEDs, and this functionality would be achievable only with organic semiconductors.

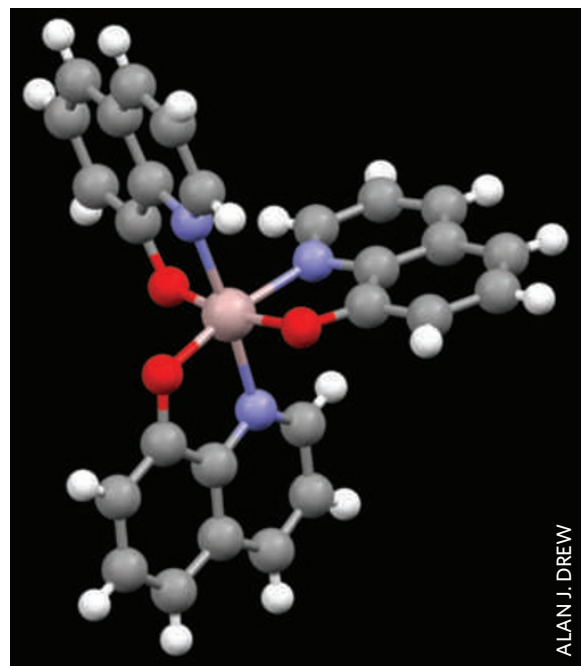

$\mathrm{Alq}_{3}$ is one of the most widely investigated organic semiconductors for both OLED and spintronic applications.

Other types of application could arise from OMAR. Markus Wohlgenannt, among the authors who discovered the effect in 2004, is actively working to understand the mechanism at the origin of this phenomenon. Furthermore, he suggests that the effect could be used in touch-screen devices. His idea is to couple an OLED-based display with a magnetic pen, generating a small magnetic field. When the pen approaches a specific pixel, the organic semiconductor detects the magnetic field through a change in resistance, which can then be converted into pixel illumination ${ }^{6}$. Recent experiments on the compound $\mathrm{Alq}_{3}$ - which is also widely investigated for OLEDs - show that an enhancement of current of up to $400 \%$ can be obtained with a magnetic field of just $200 \mathrm{mT}$ (ref. 7). These results could be beneficial for the development of OMAR-based devices.

Overall there are several possibilities, and scientists have various ideas on how to exploit these materials for device applications. However, whether or not realistic devices will be achieved is unclear, and will rely on our understanding and consequent optimization - of the mechanisms controlling spin injection and transport. As Szulczewski, Sanvito and Coey explain in their Commentary ${ }^{8}$, both aspects are quite complex, mainly due to the effect of defect states, both at the ferromagnetic electrode/organic interface and within the organic material itself. Regarding injection, one of the main problems is that it is difficult to predict how spin-polarized carriers will transfer from the electrodes to the organic layer, simply because the energy diagrams obtained by optical measurements cannot directly be used for transport. Apart from further theoretical work, experiments with different ferromagnetic electrodes and different electrode configurations could provide important insight. In addition, the recently demonstrated techniques to directly measure the spin injection ${ }^{9,10}$ will be very important tools.

For transport, although spin-orbit interaction is negligible, it is likely that the effect of the hyperfine interaction (HFI) between electron and nuclear spins is instead quite strong. Among others, Bert Koopmans believes that this interaction is connected to both the origin of OMAR and the spin-diffusion mechanism. In other words, understanding the details of the role of HFI will be important for devices based on the intrinsic magnetoresistance of organic semiconductors and those based on the transport of injected spin-polarized carriers - such as spin valves.

Given the many open questions, it is fair to say, as most scientists in the field do, that organic spintronics is still in its infancy. But it is growing in terms of both results and the attention given to it, and we shall keep following future developments with interest.

\section{References}

1. Dediu, V., Murgia, M., Matacotta, F. C., Taliani, C. \& Barbanera, S. Solid State Commun. 122, 181-184 (2002).

2. Xiong, Z. H., Wu, D., Vardeny, Z. V. \& Shi, J. Nature 427, 821-824 (2004).

3. Francis, T. L., Mermer, O., Veeraraghavan, G. \& Wohlgenannt, M New J. Phys. 6, 185 (2004).

4. Dediu, V. A., Hueso, L. E., Bergenti, I. \& Taliani, C. Nature Mater. 8, 707-716 (2009).

5. Nature Mater. 8, 696-697 (2009).

6. http://ostc.physics.uiowa.edu/ wrg/Brochure.pdf

7. Yusoff, A. R. B. M., da Silva, W. J., Serbena, J. P. M., Meruvia, M. S. \& Hummelgen, I. A. App. Phys. Lett. 94, 253305 (2009).

8. Szulczewski, G. J., Sanvito, S. \& Coey, J. M. D. Nature Mater. 8, 693-695 (2009).

9. Drew, A. J. et al. Nature Mater. 8, 109-114 (2009).

10. Cinchetti, M. et al. Nature Mater. 8, 115-119 (2009). 